\title{
When the Good Turns Ugly: Speculating Next Steps for Digital Wellbeing Tools
}

\author{
Kelly Widdicks \\ School of Computing and Communications, Lancaster University, Lancaster, UK \\ k.v.widdicks@lancaster.ac.uk
}

\begin{abstract}
Concerns surrounding technology use in society has led to the HCI community creating tools for 'digital wellbeing'. These aim to improve users' relationships with technology, but these positively motivated tools may initiate further negative impacts for users e.g. on their privacy or autonomy. Using Pierce's speculative design concepts of 'foot-in-the-door' technologies and focusing on three common digital wellbeing features (time limits and prompts, social 'do not disturb' modes, app and service blocking), I highlight how these tools are a small step away from being used to manipulate users which could enact slow shifts in users accepting such manipulation. Through this and the discussion, I accentuate that positively motivated designs may not explicitly lead to positive interactions by default. I hope this paper will facilitate speculative design and discussion in the digital wellbeing community, to ensure that our designs continue to mitigate negative impacts from technology now and in the future.
\end{abstract}

\section{CCS CONCEPTS}

- Human-centered computing $\rightarrow$ Human computer interaction (HCI).

\section{KEYWORDS}

digital wellbeing, negative impacts, research through design, speculative design, foot-in-the-door technologies, dark design

\section{ACM Reference Format:}

Kelly Widdicks. 2020. When the Good Turns Ugly: Speculating Next Steps for Digital Wellbeing Tools. In Proceedings of the 11th Nordic Conference on Human-Computer Interaction: Shaping Experiences, Shaping Society (NordiCHI '20), October 25-29, 2020, Tallinn, Estonia. ACM, New York, NY, USA, 6 pages. https://doi.org/10.1145/3419249.3420117

\section{INTRODUCTION AND BACKGROUND}

Technology and the experiences it facilitates have, and do, shape our society in many ways. These can be perceived as positive or negative for users, society and the environment. Most recently, the technology industry has received a lot of criticism for the negative implications it can have on people. In the media, headlines have highlighted some of the harmful impacts technology can have on our wellbeing [16, 56], relationships [18], democracy [14,57] and

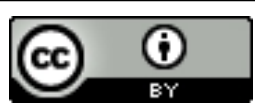

This work is licensed under a Creative Commons Attribution International 4.0 License. NordiCHI '20, October 25-29, 2020, Tallinn, Estonia

(C) 2020 Copyright held by the owner/author(s).

ACM ISBN 978-1-4503-7579-5/20/10.

https://doi.org/10.1145/3419249.3420117 privacy $[7,8,19,49]$-concerns which are seeping into consumer attitudes towards technology. In the UK, a report by Ofcom revealed $59 \%$ of UK users believe internet benefits outweigh the risks, yet eight out of ten adults in 2019 have concerns about internet usage [50]. In Europe, the Center for the Governance of Change exposed in 2019 that $70 \%$ of Europeans think technologies will bring more harm than good to society in the next decade if not controlled [25].

In the USA, the Pew Research Center uncovered users' privacy concerns over companies using their data, with $81 \%$ of Americans believing data collection risks outweigh the benefits [5]. They also found that $68 \%$ of health experts in America believe users' wellbeing will be improved or maintained from digital technologies, yet $32 \%$ believe technologies will do more harm due to issues such as: digital addiction (due to technology adopting habit-forming designs), digital deficits (e.g. on memory, focus, thinking), digital distrust (e.g. driving doubt and divisions), digital duress (e.g. rising stress, anxiety, sleeplessness) and digital dangers (e.g. to human interaction, democracy, jobs) [2].

Human-Computer Interaction (HCI) researchers are in a unique position to understand and mitigate the negative impacts of technology, given the field crosses the boundaries of technology design and users' experiences. HCI researchers have therefore offered tools and concepts to envision a better relationship between society and technology, and alleviate the negative impacts [e.g. 15, 28, 42]. Most recently, there has been a focus on designing for 'digital wellbeing' $[17,48]$. Much of this work specifically focuses on understanding or mitigating digital overuse and addiction [6, 20, 38, 40,59], and digital tools have become publicly available to users to help them better control their technology use (e.g. apps such as Forest [26] and Hold [34], Apple's screen time [4], Android's digital wellbeing [3]). Rofferello and De Russis found that such applications include selfmonitoring tools (e.g. usage tracking and data visualisations) and interventions (e.g. phone and app timers or blockers) [48]; Lyngs et al. similarly categorise 'digital self-control tools' into themes of self-tracking, service blocking or removal, goal-setting, and reward or punishment mechanisms [45].

Yet Widdicks and Pargman have criticised such design exploration for potentially enabling more control over users' online service and digital device use [62]. Lee et al. [41] have also called for researchers and practitioners to consider the negative impacts of 'positive computing' [15] systems that aim to improve users' health and wellbeing-a theme for which digital wellbeing tools are a part. Lee et al. outlined potential negative impacts that could arise such as technology dependence, privacy of user data and other ethical issues-explicitly noting that these systems could be used to manipulate individual behaviour (e.g. for product sales) [41]. Autonomy is an important aspect to wellbeing [52], and Burr et al. have pointed 
out that fear of lost self-determination should not prevent the development of technology interventions [13]; we should, therefore, not discontinue the digital wellbeing effort in HCI. However, we have a duty to ensure that what we design and develop to improve digital wellbeing does exactly that: improve digital wellbeing. But how do we mitigate against the negative impacts that might arise from an area of computing research which explicitly aims to be positive? How do we ensure what we design today, is 'future-proofed' for arising issues going forward?

HCI research in speculative design [e.g. 22, 53, 55, 63] offers a way of imagining - and re-imagining - the futures of technology. As a mode of research through design, it provides a way to "develop critical dialogues and discourse about new, alternative and future paradigms of technology use" [23, p. 5386] and incorporates ideas of 'design fiction' $[9,10,43,63]$ that can emphasise reflection on design consequences [44]-engaging "audiences in considerations of what might be" [22, p. 109]. It is therefore a useful mechanism for positioning the future impacts our designs may introduce. In this notion, as well as building upon prior work on design-led inquiries [54], Pierce introduced key vocabularly in 2019 to help HCI researchers foreground the social and ethical issues of computing-offering a way of speculating future trends and issues so that we can introduce interventions or take other design-orientated responses [53]. One of the terms Pierce introduced was the concept of "foot-in-the-door" devices [53]:

"Foot-in-the-door devices are product and services with
functional offerings and affordances that work to nor-
malize and integrate a technology, thus laying ground-
work for future adoption of features that might earlier
have been rejected as unacceptable or unnecessary. The
concept of a foot-in-the-door device emphasizes design
as practice of persuasion, manipulation, and compli-
ance." [53, p. 11]

Pierce notes that these 'foot-in-the-door' devices enable technologies to be accepted "through a combination of incremental changes (small steps) and gradual changes (slow shifts)" [53, p. 8]. In this short paper, I utilise Pierce's vocabularly to position popular digital wellbeing tools as 'foot-in-the-door' technologies. Drawing upon digital wellbeing tools available to the public, as well as HCI research in this area, I emphasise the potentially negative ethical issues that digital wellbeing tools could 'lay the groundwork for'-specifically features which put user autonomy in question. This involves applying Pierce's "speculative foot-in-the-door roadmaps/scenarios" technique [53] to anticipate future issues surrounding digital wellbeing design; in this case, imagining scenario-based changes to common digital wellbeing features - i.e. 'small steps' - which could enact negative impacts and 'slow shifts' in how these impacts, and technology itself, are perceived by users over time. These scenarios are indeed only speculative, but are offered to facilitate discussion and change in the $\mathrm{HCI}$ and digital wellbeing community by illuminating: how even the tools we design to mitigate the negative impacts of technology could inadvertently help drive them in the future; and how we might build upon speculative design to alleviate potential risks going forward. I hope that this will help the community move in a direction whereby digital wellbeing tools continue to reduce the harm that digital technology can introduce to our lives.

\section{DIGITAL WELLBEING TOOLS: SPECULATIVE SCENARIOS}

To position digital wellbeing tools as 'foot-in-the-door' technologies [53], I focus on three digital wellbeing tool features: 1) time limits and prompts, designed to shorten the length of time in which users interact with a digital device or service; 2) social 'do not disturb' modes, designed to enact periods of technology-free time to focus users' attention on face-to-face relationships; and 3) app and service blocking, designed to prevent use or overuse of an online application e.g. at specific times of the day. A speculative design scenario (specifically a 'small step' design change to current digital wellbeing tools and research) is provided for each feature to show how digital wellbeing - a movement which has the explicit motivation of creating better, or more positive, relationships between users and devices - could potentially add to the negative ethical issues arising from technology and normalise these over time ('slow shifts'). These features were selected as they are common interventions provided in digital wellbeing tools [cf. 48] and HCI literature, and are therefore useful for an initial exploration of speculating future issues from such positively motivated technologies.

\subsection{Time limits and prompts}

To encourage users to spend less time online and on their devices, digital wellbeing tools often involve time-related tracking and management. A common feature of this type involves the user being able to set usage time limits or 'goals' on specific services and devices. For example, Apple's 'Screen Time' enables users to set limits on the use of app categories, such as 30 minutes on social media apps [4]; once this limit is reached, social media apps look to be inaccessible but users are still able to access the apps by ignoring or deleting the limit. Warnings of remaining time are provided to the user through notifications-similar to how 'take a break' prompts in digital wellbeing tools work. The latter monitors the time a user spends on a service, prompting them 'in the moment' of an interaction to end their current usage session after a given period of time. For example, YouTube users have the ability to set 'take a break' reminders at a given frequency as they spend time watching videos [32].

Both of these examples attempt to engage users in reflections of their use, suggesting their time might be better spent elsewhere. However, users still have the ability to ignore the limit or prompt. In 2019, Kim et al. explored different strengths of user-defined limits on smartphone use: 1) a non-lockout tool, whereby users were only notified of their time limit goal; 2) a weak-lockout tool, whereby users would be locked out of their smartphone for an increasing period of time once a time limit goal was met; and 3) a stronglockout tool, whereby users could not access their device after their time limit [37]. Whilst the strong-lockout tool was the most effective in reducing smartphone use, in some cases participants would wait until midnight (when the limit would reset) to use their device again and participants actually found the weak-lockout tool most favourable [37].

2.1.1 Speculative scenario: 'Watch ads to dismiss'. Given popular applications and operating systems are already employing time limits and prompts, and that HCI researchers are looking into more 
restrictive ways of incorporating these, it is becoming increasingly 'normal' for these designs to exist. Yet this feature could easily become the foot-in-the-door [53] of gaining more control over users' access to services. This is most plausible with services which offer a monetarily free service to a user, at the expense of their data or their engagements in advertisements. For example, in the case of the YouTube, their prompt currently states: "Time to take a break? You've been watching for 5 minutes. Adjust or turn off this reminder in Settings." (with the options to dismiss the prompt or go to Settings) [32]. Given the platform is funded by adverts, a small step from this is the inability to dismiss the take a break prompt without engaging in some form of advertisement: "Time to take a break? You've been watching for 5 minutes. If you want to continue watching, you can dismiss this prompt by watching 3 minutes of advertisements". Here the advert ('small step') could be seen as a "design friction" or "microboundary" to use [21], disguised as something to help the user whilst inevitably exploiting theman exploitation and manipulation they may begin to accept ('slow shift') for the 'good' of their digital wellbeing.

\subsection{Social 'do not disturb' modes}

To decrease social interruptions from technology, digital wellbeing tools include features that aim to help users disengage from their devices so that they can fully concentrate on a social interaction with a friend, family member or colleague. For example, the 'Forest' app [26] assists users to "stay focused, be present" in social situations by connecting friends' devices and growing virtual trees together. Each device connected will display progress of the trees growing, keeping other apps on the device inaccessible-yet if someone uses their device for something other than Forest, the shared trees will die. This draws on the social influences of maintaining the forest for your friends. In a similar vein, Google Pixel phones now include 'Flip to Shhh' gestures [31]. By the user actively placing their device face down on a surface, the device 'do not disturb' mode will be immediately activated. This means that the devices will automatically mute sound or vibrations from messages, events, reminders or calls [30]. Google introduced this gesture to minimise users' device use whilst in the presence of others and ensure users fully appreciate their time with in-person connections [29].

In HCI research, similar social interventions have been studied such as Ko et al.'s 'Lock n' Lol' app for minimising smartphone distractions during group activities [39]. Building on this work, Park et al. look to minimise smartphone distractions whilst also ensuring users do not feel anxious about missing important notifications [51]. They developed a tool to explicitly discover specific socially-driven 'breakpoints' to deliver user notifications as friends sit together: 1) when a user is alone; 2) when a group member uses their smartphone; 3) when a user leaves the group; and 4) when the group does not converse for longer than 5 seconds [51].

2.2.1 Speculative scenario: 'Device breaks'. Whilst these tools help create dedicated time for social experiences [26, 31, 39] or utilise social cues for notification delivery [51], they make it more acceptable for technical mechanisms to be deployed during social situations and are the foot-in-the-door [53] for technology to create dedicated time for device use. Imagine the scenario of friends at dinner who have all utilised one of these tools to turn off distractions from their devices. Whilst the friends are engaging in their in-person interactions, their devices are sharing their notification data with one another. Once all users have enough notifications, the devices declare an appropriate time for a group 'device break'. The social experience is therefore interrupted by a cooperative sound from the devices to indicate all users should take some time to catch-up with the online world. Such a decision steps from being driven by the social situation (as like Park et al.'s breakpoint tool [51]) to the demands of internet-connected technology. Here the device breaks ('small step') could be seen to help users better co-ordinate their physical and online interactions, yet perhaps increasing users' dependence on technology for understanding and handling social norms-enabling new, acceptable management roles for technology and ways of enacting compliance ('slow shift') for the 'good' of users' digital wellbeing.

\subsection{App and service blocking}

To avoid distractions from specific applications or services, digital wellbeing tools now include mechanisms which allow users to block their access to these interruptive technologies. 'Freedom' and 'StayFocusd' offer opportunities to block internet access, applications and websites across multiple types of devices (Windows, Mac, Android, iOS and Chrome)-helping users to "focus on your work, break a habit, or simply improve your relationship with technology" [27]. Such features are also offered on popular mobile operating systems: Apple's 'Downtime' feature allows users to only grant access to specific apps during a given period [4] and Android's 'Focus mode' similarly enables users to pause access to distracting apps [3]. Android additionally offers a 'Work profile' to "Leave work at the office", meaning work-related apps (e.g. email, calendar) can be made less accessible outside of working hours [3]. Arguing that other website blockers are easy to ignore, the application 'Cold Turkey' ensures that no software blocking session can be undone by default [60].

$\mathrm{HCI}$ researchers have been exploring these technologies and their effect on work productivity by blocking applications or services whilst users are working. Investigations have included blocking apps at specific times [59], across multiple devices [36], or during the full working day for sites users find distracting [46]. Such explorations have been carried out with different participant demographics (e.g. students, workplaces), but all allow users to have the choice in listing specific applications or services they themselves find distracting. This ensures a tailored approach that helps mitigate users' personal workplace interruptions.

2.3.1 Speculative scenario: 'Work-controlled profiles'. Currently, software which provides users with the opportunity to block access to online services ensures that control resides with the userhelping people to stay productive for themselves. Yet, these tools could be the foot-in-the-door [53] technology for companies to utilise similar applications to monitor employees, particularly as people are given more freedom to work from home (or required to, as with the COVID-19 pandemic). What if there was a shift in blocking control from users to employers, with 'Work profiles' on devices potentially becoming 'Work-controlled profiles'? Such profiles would provide an opportunity for the employer to block access to sites they want to limit their employees from accessing during work hours. Here employers could argue that employees utilising 
these profile tools ('small step') will be less distracted and perhaps more likely to be promoted by showing work dedication, but in fact such tools offer new opportunities to monitor and control staffopening up new ways of acceptably, and persuasively, surveilling users ('slow shift') for the 'good' of their digital wellbeing.

\section{DISCUSSION AND CONCLUSIONS}

In this short paper, I have explored features of digital wellbeing tools as foot-in-the-door technologies: explicitly foregrounding such "design as practice of persuasion, manipulation, and compliance" [53, p. 11] to emphasise the potential negative impacts that these positively motivated technologies could enable or normalise. The speculative scenarios provided offer examples of what could happen next in the design of digital wellbeing tools. Whether design changes in digital wellbeing tools will really enable users to be exploited through future advertisement targeting, managed socially by technology, or surveilled at work by companies, is yet to be defined in the near future. For these speculative designs to become apparent and significant, they would depend on: a) the actions of different actors (e.g. HCI designers, service providers, technologists, employers) to initially develop the 'small steps' [53]; and b) the development of 'slow shifts' [53] which lead to users' accepting the design.

HCI designers and practitioners would in fact have to promote tools for digital wellbeing whilst turning these 'persuasive designs' [24] against users-a direction which heads towards 'dark design' patterns [cf. 11, 12, 33, 47, 64]. Cox et al. argue that 'microboundaries' (e.g. to service or device use) differ to dark design patterns as they are used to support users, rather than act against them [21]. However, through participatory design workshops creating interventions to reduce device and service use (often mimicking digital wellbeing tools), Widdicks [61] has found stronger links to deceptive design in this space-suggesting dark patterns could be used 'for good' [61] as a form of 'benevolent deception' [1]. This is the grey area whereby the actors in point a) above could find themselves, potentially leading to the endorsement of these speculative 'small steps' [53] in digital wellbeing. This is also concerning for point b), for two reasons: 1 ) dark patterns could be portrayed in a good light to users for their digital wellbeing, creating easier acceptance of manipulative designs; and 2) the participant discussion of dark patterns 'for good' [61] may already indicate the presence of 'slow shifts' [53] in users' acceptance of what such technology is, or is not, 'allowed' to do-enabling the "future adoption of features that might earlier have been rejected as unacceptable or unnecessary" [53, p. 11].

This highlights the importance of designing digital wellbeing tools which are truly positive. As I have shown in this paper, digital wellbeing tools are only small steps from being used in manipulative forms disguised as positive actions and may enable slow shifts in acceptance for what technology should do or be [53]. The technology sector moves at an innovation rate so fast that we, as $\mathrm{HCI}$ designers and practitioners, do not have the time to really reflect on how our innovations might be used or pave the way for future interactions. This is reiterated with the digital wellbeing movement in the last few years, with the process being described as a "race towards digital wellbeing" [48]. But we need to remember that these tools are unique: they can affect users' interactions with all different types technologies, and they can be advertised to positively improve users' relationships with technology. In a similar vein to Spiel et al.'s work on fitness trackers, digital wellbeing tools have the ability to employ "emotional design tricks to engage in a progressive re-definition of what it means to be human" [58, p. 4]. Therefore the HCI community should not be 'racing' in this; we should be exploring all potential avenues of impacts that digital wellbeing tools may introduce now and in the future. The simple fact that these tools are positively motivated does not mean they will create positive interactions by default.

I want to express that this paper is not set to undermine the great work of the digital wellbeing community. I am too part of that research, and aim to continue my work in this domain. However, it is through this involvement that I have developed these reflections, and I see this short paper as a way to remind the digital wellbeing community - including myself - how even the tools we design to mitigate the negative impacts of technology could inadvertently help drive them. The very notion that digital wellbeing tools offer ways of restricting use, may act as a 'foot-in-the-door' [53] for further manipulative designs which I'm sure no one in the digital wellbeing community would want to witness. What we need is to investigate all impacts of digital wellbeing tools, and look to ways of 'future-proofing' these designs so that they cannot act as 'foot-in-the-door' technologies. Pierce's speculative foot-in-thedoor roadmaps/scenarios [53] as used in this paper offer one way of formulating future designs to avoid and mitigate against. We may also look to build upon speculative or design fiction research more generally [e.g. 9, 23] and further bridge the temporal gap between design and ethnography [35], to develop methodologies which merge speculative designs with longer term participatory studies-aiming to probe and evaluate how users' acceptance of speculative digital wellbeing designs may change over time. How that may happen is beyond the scope of this paper, but I call for the digital wellbeing community to engage in speculative work to help think more deeply about unforeseen impacts of our tools. We can embrace these complexities in designing for digital wellbeing, and continue moving in a direction whereby our designs reduce the harm that digital technology can introduce to our lives now and in the future.

\section{ACKNOWLEDGMENTS}

Thank you to the EPSRC (grant no. EP/R513076/1). Thank you to the reviewers, as well as Oliver Bates, Bran Knowles, Carolynne Lord, Christian Remy and Vanessa Thomas for all their encouragement, support and valuable feedback.

\section{REFERENCES}

[1] Eytan Adar, Desney S. Tan, and Jaime Teevan. 2013. Benevolent Deception in Human Computer Interaction. In Proceedings of the SIGCHI Conference on Human Factors in Computing Systems (Paris, France) (CHI '13). Association for Computing Machinery, New York, NY, USA, 1863-1872. https://doi.org/10.1145/ 2470654. 2466246

[2] Janna Anderson and Lee Rainie. 2018. The Future of Well-Being in a Tech-Saturated World. Pew Research Centre (2018). https: //www. pewinternet.org/2018/04/17/the-future-of-wellbeing-in-a-tech-saturated-world/, accessed February 2020.

[3] Android. 2020. Digital Wellbeing: New ways to find balance for you and your family. https://www. android.com/digital-wellbeing/, accessed 
February 2020.

[4] Apple. 2018. iOS 12 introduces new features to reduce interruptions and manage Screen Time. Apple Newsroom (2018). https://www.apple.com/uk/new sroom/2018/06/ios-12-introduces-new-features-to-reduceinterruptions-and-manage-screen-time/, accessed February 2020.

[5] Brooke Auxier, Lee Rainie, Monica Anderson, Andrew Perrin, Madhu Kumar, and Erica Turner. 2019. Americans and Privacy: Concerned, Confused and Feeling Lack of Control Over Their Personal Information. Pew Research Centre (2019). https: //www. pewresearch.org/internet/2019/11/15/americ ans-and-privacy-concerned-confused-and-feeling-lack-ofcontrol-over-their-personal-information/, accessed February 2020

[6] Eric P. S. Baumer, Shion Guha, Patrick Skeba, and Geraldine Gay. 2019. All Users Are (Not) Created Equal: Predictors Vary for Different Forms of Facebook Non/Use. Proc. ACM Hum.-Comput. Interact. 3, CSCW, Article 80 (Nov. 2019), 28 pages. https://doi.org/10.1145/3359182

[7] The BBC. 2019. Data surveillance powers unlawfully wide, court told. $B B C$ News (2019). https://www.bbc.co.uk/news/uk-48663613, accessed February 2020

[8] The BBC. 2020. What are the rules on workplace surveillance? BBC News (2020) https://www.bbc.co.uk/news/explainers-51571684, accessed February 2020 .

[9] Mark Blythe. 2014. Research Through Design Fiction: Narrative in Real and Imaginary Abstracts. In Proceedings of the SIGCHI Conference on Human Factors in Computing Systems (Toronto, Ontario, Canada) (CHI '14). ACM, New York, NY, USA, 703-712. https://doi.org/10.1145/2556288.2557098

[10] Mark Blythe, Jamie Steane, Jenny Roe, and Caroline Oliver. 2015. Solutionism, the Game: Design Fictions for Positive Aging. In Proceedings of the 33rd Annual ACM Conference on Human Factors in Computing Systems (Seoul, Republic of Korea) (CHI '15). Association for Computing Machinery, New York, NY, USA, 3849-3858. https://doi .org/10.1145/2702123.2702491

[11] Harry Brignull. 2011. Dark Patterns: Deception vs. Honesty in UI Design. Interaction Design, Usability 338 (2011)

[12] Harry Brignull. 2019. Dark Patterns. https://www.darkpatterns.org/ accessed April 2020.

[13] Christopher Burr, Mariarosaria Taddeo, and Luciano Floridi. 2019. The Ethics of Digital Well-Being: A Thematic Review. Science and Engineering Ethics (2019), $1-31$

[14] Carole Cadwalladr and Emma Graham-Harrison. 2018. Revealed: 50 million Facebook profiles harvested for Cambridge Analytica in major data breach. The Guardian (2018). https://www. theguardian.com/news/2018/mar/ 17 /cambridge-analytica-facebook-influence-us-election, accessed February 2020.

[15] Rafael A Calvo and Dorian Peters. 2014. Positive computing: technology for wellbeing and human potential. MIT Press.

[16] Denis Campbell. 2019. Children aged five and under at risk of internet addiction - Barnardo's. The Guardian (2019). https: //www. theguardian.com/society/2019/jun/11/children-agedfive-and-under-at-risk-of-internet-addiction-barnardos, accessed February 2020

[17] Marta E. Cecchinato, John Rooksby, Alexis Hiniker, Sean Munson, Kai Lukoff, Luigina Ciolfi, Anja Thieme, and Daniel Harrison. 2019. Designing for Digital Wellbeing: A Research \& Practice Agenda. In Extended Abstracts of the 2019 CHI Conference on Human Factors in Computing Systems (Glasgow, Scotland Uk) (CHI $E A$ '19). Association for Computing Machinery, New York, NY, USA, Article W17, 8 pages. https://doi.org/10.1145/3290607.3298998

[18] Stella Chatzitheochari and Killian Mullan. 2019. Alone together: how mobile devices have changed family time. The Conversation (2019). https://theconversation.com/alone-together-how-mobiledevices-have-changed-family-time-111478?utm_medium=Soc ial\&utm_source=Twitter\#Echobox $=1552402817$, accessed February 2020.

[19] Brian X. Chen. 2020. Your Doorbell Camera Spied on You. Now What? The New York Times (2020). https: //www. nytimes.com/2020/02/19/technolo gy/personaltech/ring-doorbell-camera-spying.html, accessed February 2020

[20] Justin Cheng, Moira Burke, and Elena Goetz Davis. 2019. Understanding Perceptions of Problematic Facebook Use: When People Experience Negative Life Impact and a Lack of Control. In Proceedings of the 2019 CHI Conference on Human Factors in Computing Systems (Glasgow, Scotland UK) (CHI '19). Association for Computing Machinery, New York, NY, USA, Article 199, 13 pages. https://doi.org/10.1145/3290605.3300429

[21] Anna L. Cox, Sandy J.J. Gould, Marta E. Cecchinato, Ioanna Iacovides, and Ian Ren free. 2016. Design Frictions for Mindful Interactions: The Case for Microboundaries. In Proceedings of the 2016 CHI Conference Extended Abstracts on Human Factors in Computing Systems (San Jose, California, USA) (CHI EA '16). ACM, New York, NY, USA, 1389-1397. https://doi.org/10.1145/2851581.
2892410

[22] Carl DiSalvo. 2012. FCJ-142 Spectacles and tropes: Speculative design and contemporary food cultures. The Fibreculture fournal 20 2012: Networked Utopias and Speculative Futures (2012).

[23] Chris Elsden, David Chatting, Abigail C. Durrant, Andrew Garbett, Bettina Nissen, John Vines, and David S. Kirk. 2017. On Speculative Enactments. In Proceedings of the 2017 CHI Conference on Human Factors in Computing Systems (Denver, Colorado, USA) (CHI '17). Association for Computing Machinery, New York, NY, USA, 5386-5399. https://doi.org/10.1145/3025453.3025503

[24] Brian J Fogg. 2009. A behavior model for persuasive design. In Proceedings of the 4th international Conference on Persuasive Technology. ACM, 40.

[25] Centre for The Governance of Change. 2019. European Tech Insights 2019: Mapping European Attitudes to Technological Change and its Governance. Technical Report. IE University. https://docs.ie.edu/cgc/European-Tech-Insigh ts-2019. pdf, accessed February 2020.

[26] Forest. 2020. https://www. forestapp.cc/, accessed February 2020.

[27] Freedom. 2020. https://freedom. to/stayfocusd/, accessed February 2020.

[28] Batya Friedman, Peter H Kahn, and Alan Borning. 2008. Value sensitive design and information systems. The handbook of information and computer ethics (2008), 69-101.

[29] Google. 2020. Digital Wellbeing: Minimize distractions https://we llbeing.google/get-started/minimize-distractions/, accessed February 2020.

[30] Google. 2020. Pixel Phone Help: Limit interruptions with Do Not Disturb on Pixel phone. https://support.google.com/pixelphone/answer $/ 6111295$ ?hl=en, accessed February 2020.

[31] Google. 2020. Pixel Phone Help: Use gestures on your device. Flip to Shhh: https://support.google.com/pixelphone/answer/7443425\#fl ip_to_shhh, accessed February 2020.

[32] Google. 2020. YouTube Help: Take a break reminder. https: //support.google.com/youtube/answer/9012523?co=GENIE .Plat form\%3DAndroid\&hl=en\&oco=0, accessed February 2020.

[33] Colin M. Gray, Yubo Kou, Bryan Battles, Joseph Hoggatt, and Austin L. Toombs. 2018. The Dark (Patterns) Side of UX Design. In Proceedings of the 2018 CHI Conference on Human Factors in Computing Systems (Montreal QC, Canada) (CHI '18). Association for Computing Machinery, New York, NY, USA, Article 534, 14 pages. https://doi.org/10.1145/3173574.3174108

[34] Hold. 2020. https://www.hold.app/, accessed April 2020.

[35] Vera Khovanskaya, Phoebe Sengers, Melissa Mazmanian, and Charles Darrah. 2017. Reworking the Gaps between Design and Ethnography. In Proceedings of the 2017 CHI Conference on Human Factors in Computing Systems (Denver, Colorado, USA) (CHI '17). Association for Computing Machinery, New York, NY, USA, 5373-5385. https://doi.org/10.1145/3025453.3026051

[36] Jaejeung Kim, Chiwoo Cho, and Uichin Lee. 2017. Technology Supported Behavior Restriction for Mitigating Self-Interruptions in Multi-Device Environments. Proc. ACM Interact. Mob. Wearable Ubiquitous Technol. 1, 3, Article 64 (Sept. 2017), 21 pages. https://doi.org/10.1145/3130932

[37] Jaejeung Kim, Hayoung Jung, Minsam Ko, and Uichin Lee. 2019. GoalKeeper: Exploring Interaction Lockout Mechanisms for Regulating Smartphone Use. Proc. ACM Interact. Mob. Wearable Ubiquitous Technol. 3, 1, Article 16 (March 2019), 29 pages. https://doi.org/10.1145/3314403

[38] Jaejeung Kim, Joonyoung Park, Hyunsoo Lee, Minsam Ko, and Uichin Lee. 2019. LocknType: Lockout Task Intervention for Discouraging Smartphone App Use. In Proceedings of the 2019 CHI Conference on Human Factors in Computing Systems (Glasgow, Scotland UK) (CHI '19). ACM, New York, NY, USA, Article 697, 12 pages. https://doi.org/10.1145/3290605.3300927

[39] Minsam Ko, Seungwoo Choi, Koji Yatani, and Uichin Lee. 2016. Lock n'LoL: group-based limiting assistance app to mitigate smartphone distractions in group activities. In Proceedings of the 2016 CHI Conference on Human Factors in Computing Systems. 998-1010.

[40] Simone Lanette, Phoebe K. Chua, Gillian Hayes, and Melissa Mazmanian. 2018. How Much is 'Too Much'?: The Role of a Smartphone Addiction Narrative in Individuals' Experience of Use. Proceedings of the ACM on Human-Computer Interaction 2, CSCW, Article 101 (Nov 2018), 22 pages. https://doi.org/ $10.1145 / 3274370$

[41] Uichin Lee, Kyungsik Han, Hyunsung Cho, Kyong-Mee Chung, Hwajung Hong, Sung-Ju Lee, Youngtae Noh, Sooyoung Park, and John M. Carroll. 2019. Intelligent positive computing with mobile, wearable, and IoT devices: Literature review and research directions. Ad Hoc Networks 83 (2019), 8 - 24. https://doi.or g/10.1016/j.adhoc. 2018.08 .021

[42] Hanlin Li, Bodhi Alarcon, Sara Milkes Espinosa, and Brent Hecht. 2018. Out of Site: Empowering a New Approach to Online Boycotts. Proc. ACM Hum.-Comput. Interact. 2, CSCW, Article 106 (Nov. 2018), 28 pages. https://doi.org/10. $1145 / 3274375$ 
[43] Joseph Lindley and Paul Coulton. 2015. Back to the Future: 10 Years of Design Fiction. In Proceedings of the 2015 British HCI Conference (Lincoln, Lincolnshire, United Kingdom) (British HCI '15). Association for Computing Machinery, New York, NY, USA, 210-211. https://doi .org/10.1145/2783446. 2783592

[44] Conor Linehan, Ben J. Kirman, Stuart Reeves, Mark A. Blythe, Joshua G. Tanenbaum, Audrey Desjardins, and Ron Wakkary. 2014. Alternate Endings: Using Fiction to Explore Design Futures. In $\mathrm{CHI}$ '14 Extended Abstracts on $\mathrm{Hu}$ man Factors in Computing Systems (Toronto, Ontario, Canada) (CHI EA '14) Association for Computing Machinery, New York, NY, USA, 45-48. https: //doi.org/10.1145/2559206.2560472

[45] Ulrik Lyngs, Kai Lukoff, Petr Slovak, Reuben Binns, Adam Slack, Michael Inzlicht, Max Van Kleek, and Nigel Shadbolt. 2019. Self-Control in Cyberspace: Applying Dual Systems Theory to a Review of Digital Self-Control Tools. In Proceedings of the 2019 CHI Conference on Human Factors in Computing Systems (Glasgow, Scotland Uk) (CHI '19). Association for Computing Machinery, New York, NY, USA, Article 131, 18 pages. https://doi.org/10.1145/3290605.3300361

[46] Gloria Mark, Mary Czerwinski, and Shamsi T. Iqbal. 2018. Effects of Individual Differences in Blocking Workplace Distractions. In Proceedings of the 2018 CHI Conference on Human Factors in Computing Systems (Montreal QC, Canada) (CHI '18). Association for Computing Machinery, New York, NY, USA, Article 92 , 12 pages. https://doi.org/10.1145/3173574.3173666

[47] Arunesh Mathur, Gunes Acar, Michael J. Friedman, Elena Lucherini, Jonathan Mayer, Marshini Chetty, and Arvind Narayanan. 2019. Dark Patterns at Scale: Findings from a Crawl of $11 \mathrm{~K}$ Shopping Websites. Proc. ACM Hum.-Comput. Interact. 3, CSCW, Article 81 (Nov. 2019), 32 pages. https://doi.org/10. $1145 / 3359183$

[48] Alberto Monge Roffarello and Luigi De Russis. 2019. The Race Towards Digital Wellbeing: Issues and Opportunities. In Proceedings of the 2019 CHI Conference on Human Factors in Computing Systems (Glasgow, Scotland Uk) (CHI '19). Association for Computing Machinery, New York, NY, USA, Article 386, 14 pages. https://doi.org/10.1145/3290605.3300616

[49] Sky News. 2020. Live facial recognition technology used by police at Oxford Circus. (2020). https://news. sky.com/story/live-facial-recognit ion-technology-used-by-police-at-oxford-circus-11938683, accessed February 2020.

[50] Ofcom. 2019. Online Nation 2019 report. Technical Report. Ofcom. https://www.ofcom.org.uk/__data/assets/pdf_file/ 0024/149253/online-nation-summary.pdf, accessed February 2020

[51] Chunjong Park, Junsung Lim, Juho Kim, Sung-Ju Lee, and Dongman Lee. 2017. Don't Bother Me. I'm Socializing! A Breakpoint-Based Smartphone Notification System. In Proceedings of the 2017 ACM Conference on Computer Supported Cooperative Work and Social Computing (Portland, Oregon, USA) (CSCW '17). Association for Computing Machinery, New York, NY, USA, 541-554. https : //doi.org/10.1145/2998181.2998189

[52] Dorian Peters, Rafael A Calvo, and Richard M Ryan. 2018. Designing for motivation, engagement and wellbeing in digital experience. Frontiers in Psychology 9 (2018), 797.

[53] James Pierce. 2019. Smart Home Security Cameras and Shifting Lines of Creepi ness: A Design-Led Inquiry. In Proceedings of the 2019 CHI Conference on $\mathrm{Hu}$ man Factors in Computing Systems (Glasgow, Scotland Uk) (CHI '19). Association for Computing Machinery, New York, NY, USA, Article 45, 14 pages. https://doi.org/10.1145/3290605.3300275

[54] James Pierce and Carl DiSalvo. 2018. Addressing Network Anxieties with Alternative Design Metaphors. In Proceedings of the 2018 CHI Conference on Human Factors in Computing Systems (Montreal QC, Canada) (CHI '18). Association for Computing Machinery, New York, NY, USA, Article 549, 13 pages. https://doi.org/10.1145/3173574.3174123

[55] Jon Rogers, Loraine Clarke, Martin Skelly, Nick Taylor, Pete Thomas, Michelle Thorne, Solana Larsen, Katarzyna Odrozek, Julia Kloiber, Peter Bihr, and et al 2019. Our Friends Electric: Reflections on Advocacy and Design Research for the Voice Enabled Internet. In Proceedings of the 2019 CHI Conference on Human Factors in Computing Systems (Glasgow, Scotland UK) (CHI '19). Association for Computing Machinery, New York, NY, USA, Article 114, 13 pages. https: //doi.org/10.1145/3290605.3300344

[56] Haroon Siddique. 2019. One in eight UK adults have suicidal thoughts over body image - poll. The Guardian (2019). https: //www. theguardian.com/lifeandstyle/2019/may/13/bodyimage-survey-one-in-eight-uk-adults-suicidal-thoughts, accessed February 2020.

[57] Sabrina Siddiqui. 2019. Half of Americans see fake news as bigger threat than terrorism, study finds. The Guardian (2019). https://www. theguardia n.com/us-news/2019/jun/06/fake-news-how-misinformationbecame-the-new-front-in-us-political-warfare, accessed February 2020.
[58] Katta Spiel, Fares Kayali, Louise Horvath, Michael Penkler, Sabine Harrer, Miguel Sicart, and Jessica Hammer. 2018. Fitter, Happier, More Productive? The Normative Ontology of Fitness Trackers. In Extended Abstracts of the 2018 CHI Conference on Human Factors in Computing Systems (Montreal QC, Canada) (CHI EA '18). Association for Computing Machinery, New York, NY, USA, Article alt08, 10 pages. https://doi.org/10.1145/3170427.3188401

[59] Vincent W.-S. Tseng, Matthew L. Lee, Laurent Denoue, and Daniel Avrahami. 2019. Overcoming Distractions during Transitions from Break to Work Using a Conversational Website-Blocking System. In Proceedings of the 2019 CHI Conference on Human Factors in Computing Systems (Glasgow, Scotland UK) (CHI '19). Association for Computing Machinery, New York, NY, USA, Article 467, 13 pages. https://doi.org/10.1145/3290605.3300697

[60] Cold Turkey. 2019. https://getcoldturkey.com/, accessed February 2020.

[61] Kelly Widdicks. 2020. Understanding and Mitigating the Impact of Internet Demand in Everyday Life. Ph.D. Dissertation. School of Computing and Communications, Lancaster University.

[62] Kelly Widdicks and Daniel Pargman. 2019. Breaking the Cornucopian Paradigm: Towards Moderate Internet Use in Everyday Life. In Proceedings of the Fifth Workshop on Computing Within Limits (Lappeenranta, Finland) (LIMITS '19). ACM, New York, NY, USA, Article 2, 8 pages. https://doi.org/10.1145/ 3338103.3338105

[63] Richmond Y. Wong and Deirdre K. Mulligan. 2016. When a Product Is Still Fictional: Anticipating and Speculating Futures through Concept Videos. In Proceedings of the 2016 ACM Conference on Designing Interactive Systems (Brisbane, QLD, Australia) (DIS '16). Association for Computing Machinery, New York, NY, USA, 121-133. https://doi.org/10.1145/2901790.2901801

[64] José P Zagal, Staffan Björk, and Chris Lewis. 2013. Dark patterns in the design of games. In Foundations of Digital Games 2013. 